\title{
Ritonavir inhibits NF-AT activation through modulation of the PI-3
} kinase/Akt Ppathway Shibani Pati ${ }^{1}$, Anh Thu Nguyen ${ }^{2}$, William Reid ${ }^{1}$, J Scott Foulke Jr ${ }^{1}$, Anjana Yadav ${ }^{1}$, Fabio Romerio ${ }^{1}$, Frank Weichold ${ }^{3}$ and Marvin Reitz*3

\author{
Address: ${ }^{1}$ Institute of Human Virology, University of Maryland Biotechnology Institute, Baltimore, Maryland, ${ }^{2}$ University of Maryland, Baltimore \\ County, USA and ${ }^{3}$ Morgan State University, Baltimore, Maryland, USA \\ * Corresponding author
}

from 2006 International Meeting of The Institute of Human Virology

Baltimore, USA. 17-2I November, 2006

Published: 21 December 2006

Retrovirology 2006, 3(SuppI I):S68 doi:10.1186/1742-4690-3-SI-S68

(c) 2006 Pati et al; licensee BioMed Central Ltd.

The HIV protease inhibitor ritonavir has activities apparently unrelated to its inhibition of the HIV protease, including anti-tumor activity in vivo and in vitro, induction of lipodystrophy in vivo, proteasome inhibition, and inhibition of NFK. Here we show that ritonavir inhibits activation of NF-AT induced by PMA plus ionomycin and by the human herpes virus- 8 chemokine receptor homologue, vGPCR. Inhibition occurred by modulation of the PI-3 kinase/Akt/GSK-3 pathway. Ritonavir treatment led to decreased Akt phosphorylation and a resultant decrease in GSK-3 phosphorylation and failed to inhibit NF-AT in GSK-3 $\beta$-/- knockout cells. Inhibition of multiple signaling pathways by ritonavir may partly explain its antitumor activities as well as other effects of ritonavir that are unrelated to its anti-retroviral activity. Taken together, the data suggest that ritonavir may have intrinsic immunomodulatory activities. This work was supported in part by grant RO1 CA099905-01 from NCI. 